\title{
Il y aurait exactement quatre linguistes qui...
}

Sur la valeur et la combinaison rare mais non exclue du conditionnel épistémique avec exactement + numéral cardinal

Il y aurait exactement quatre linguistes qui... On the Meaning and Rare but not Inexistant Combination of the Epistemic Conditional with exactement + cardinal numeral

\section{Patrick Dendale et Diana Andrei}

\section{(2) OpenEdition}

Journals

Édition électronique

URL : http://journals.openedition.org/pratiques/2673

DOI : $10.4000 /$ pratiques. 2673

ISSN : 2425-2042

Éditeur

Centre de recherche sur les médiations (CREM)

Référence électronique

Patrick Dendale et Diana Andrei, « // y aurait exactement quatre linguistes qui... », Pratiques [En ligne], 167-168 | 2015, mis en ligne le 04 avril 2016, consulté le 03 mai 2019. URL : http:// journals.openedition.org/pratiques/2673; DOI : 10.4000/pratiques.2673

Ce document a été généré automatiquement le 3 mai 2019.

(c) Tous droits réservés 


\section{Il y aurait exactement quatre linguistes}

\section{qui...}

Sur la valeur et la combinaison rare mais non exclue du conditionnel épistémique avec exactement + numéral cardinal

Il y aurait exactement quatre linguistes qui... On the Meaning and Rare but not Inexistant Combination of the Epistemic Conditional with exactement + cardinal numeral

Patrick Dendale et Diana Andrei

\section{Définition de la problématique}

1 Le point de départ de cette étude est la double constatation que le conditionnel épistémique $(\mathrm{CE})$ se rencontre très régulièrement combiné à un numéral cardinal précédé d'un modifieur de quantification approximative comme au moins, environ, près de, aux alentours de, à peu près, entre ... et ..., etc. (cf. les exemples 1-3), ou avec un collectif comme dizaine de... (cf. 4), mais qu'il est assez rare de le trouver attesté en combinaison avec un numéral précédé du modifieur exactement (cf. 5):

(1) Parmi les personnes arrêtées, il y aurait au moins 74 mineurs ${ }^{1}$.

(2) Par contre, la diaspora arménienne est relativement nombreuse en Suisse et compterait actuellement entre 4'000 et 6'000 personnes ${ }^{2}$.

(3) Il y aurait près de 50 millions de pauvres aux États-Unis.

(4) D'autres sources dans la région affirment qu'il y aurait une dizaine de morts du côté du M23 et onze capturés. (Le Potentiel online, 15/07/13, 13 h 15).

(5) Parmi les personnes arrêtées, il y aurait exactement 74 mineurs (forgé).

2 La combinaison est en tout cas une « exclue » de Frantext : une recherche de la séquence " aurait / auraient + exactement" n'a fourni aucun exemple avec un conditionnel épistémique. Les seuls exemples trouvés étaient soit des conditionnels temporels, soit des conditionnels d'éventualité. 
Une première explication de cette constatation, trop peu nuancée, comme nous le verrons, pourrait consister à penser qu'il y aurait une incompatibilité entre la valeur et/ ou la fonction du marqueur exactement et la valeur et/ou la fonction du CE. En effet, dans notre analyse, le CE est utilisé par le locuteur quand celui-ci ne peut pas ou ne veut pas prendre en charge la vérité d'une information (par exemple celle concernant les 74 personnes arrêtées dans5) alors que le marqueur exactement signalerait que l'information quantifiée est exacte, correcte et donc vraie. Il y aurait alors une contradiction entre ce que sert à marquer exactement (l'exactitude d'un chiffre et la vérité pour le locuteur de l'information chiffrée) et ce que sert à marquer le CE (la non-prise en charge de la vérité de l'information chiffrée).

Un premier problème pour cette hypothèse est que des exemples comme 5 , même s'ils sont absents de la base textuelle de Frantext, ne sont nullement inexistants dans d'autres bases textuelles. Une recherche élargie sur Internet nous a en effet fourni une petite collection d'exemples, que nous présenterons plus loin et qui montrent que la combinaison est bel et bien existante.

La question qui se pose alors est double $: 1^{\circ}$ ) pourquoi ces exemples sont-ils si rares ? $2^{\circ}$ ) comment se fait-il qu'ils soient quand même attestés, malgré les difficultés théoriques que nous venons d'évoquer?

Dans cette étude, nous allons examiner sous quelles conditions la combinaison du CE avec l'adverbe exactement + numéral cardinal est possible et quelles sont alors les caractéristiques d'exactement et du conditionnel épistémique. Notre étude nous conduira à examiner les différentes interprétations de l'adverbe exactement + numéral cardinal dans ce type de phrases et l'effet de la présence du CE sur l'interprétation de cet adverbe. Cette analyse permettra d'expliquer à la fois l'existence et la carence de phrases avec ces éléments.

Commençons par examiner la valeur d'exactement «en situation plus ou moins contrôlée », c'est-à-dire dans des phrases sans CE.

\section{La valeur d'exactement dans les phrases sans CE}

Notons tout d'abord qu'exactement est un adverbe qui se rencontre dans des distributions variées telles que celles illustrées ici, trop sommairement, par les exemples 6-8:

(6) Les meubles, que je connaissais si bien depuis seize ans, semblaient avoir été conservés sous verre, tant ils étaient exactement les mêmes (Balzac, 1830, TLFi, s.v. exactement).

(7) [Chaque parti] se voyait attribuer un nombre de sièges exactement proportionnel au total des voix recueillies par lui sur l'ensemble du territoire (De Gaulle, TLFi, s.v. exactement).

(8) Paul-Albert était exactement de mon âge (TLFi, s.v. exactement).

6 Dans ce qui suit, nous ne parlerons que des cas où, dans la distribution droite, figure un numéral cardinal, comme 9 , mais où en plus apparait un $\mathrm{CE}$ :

(9) C'était plutôt un puits qu'un escalier. Il comptait exactement quatorze marches pour chacun des étages (il y en avait trois), [...] (Genet, 1947, dans Frantext). 
7 La composition morphologique de l'adverbe exactement suggère que le sens de ce mot a à voir avec l'exactitude - dans notre cas, l'exactitude d'un chiffre. Et le mot exactitude, selon le TLFi, contient l'idée de "rigueur excluant l'approximation » (s.v. exactement). D'ailleurs, l'adverbe entre souvent en opposition paradigmatique avec l'adverbe approximativement et avec des modifieurs de quantification approximative comme environ, autour de, aux alentours de, près de... Il sélectionne une seule valeur et non une série de valeurs possibles.

En résumé, le sens d'exactement doit sans doute être défini non seulement par rapport au sens d'exactitude, mais aussi par rapport au sens de non-approximation et donc d' (im)précision.

8 Dans une étude récente de S. Adler et M. Asnes $(2014)^{3}$, les notions de précision et d' exactitude ont été définies de façon précise. Pour ces auteurs, la précision, dans un sens technique, réfère à ce «qui a une marge d'erreur nulle ou négligeable » ou " qui a un degré non élevé de dispersion des valeurs " par rapport à la valeur de référence (p. 23). L' exactitude, elle, réfère à « la conformité des résultats à une valeur vraie ou admise ». Les exemples qu'elles donnent pour illustrer l'emploi technique des termes précis/imprécis et exact/inexact permettent de discerner au moins certaines différences entre ces termes.

(10) Le locuteur sait que l'âge de Marie est de 58 ans. Il peut donner une des réponses suivantes à quelqu'un qui se renseigne sur son âge :

\begin{tabular}{|l|l|l|}
\hline a. Marie a 58 ans & précis $^{4}$ et exact \\
\hline & b. Marie a entre 55 et 60 ans & imprécis et exact \\
\hline & c. Marie a entre 45 et 50 ans & imprécis et inexact \\
\hline d. Marie a 60 ans & précis et inexact ou imprécis et exact \\
\hline e. Marie a 50 ans & précis et inexact ou imprécis et inexact ${ }^{5}$ \\
\hline
\end{tabular}

9 Si on compare ces réponses possibles, on voit qu'y est qualifiée de précise une indication de l'âge de Marie où il est fait référence à une seule valeur (a., d. et e.), et ceci indépendamment de la conformité/non-conformité de cette valeur avec la valeur de référence (58 ans) et indépendamment de la proximité de cette valeur $(58,60,50$ ans) par rapport à la valeur de référence. Sont qualifiées d'imprécises les indications chiffrées précédées d'un modifieur de quantification approximative (ici entre ... et ...), de même que les chiffres ronds $(50,60)$ dont on sait qu'ils sont arrondis. Est qualifiée d'exacte une indication chiffrée qui correspond à la valeur de référence et qui contient une indication chiffrée désignant une série de valeurs qui incluent la valeur de référence ou bien un seul chiffre. Sont qualifiées d'inexactes les indications chiffrées qui ne contiennent qu'un chiffre et qui ne correspondent pas à la valeur de référence, de même que les indications chiffrées qui désignent des suites de valeurs qui ne comprennent pas parmi les valeurs désignées la valeur de référence. L'indication chiffrée dans 10b. est qualifiée d'exacte (et, partant, la proposition où elle apparait de vraie), parce que la valeur de référence, 58, est incluse dans la suite de valeurs 55-60; cela n'est pas le cas pour 10c., où l'indication chiffrée doit être qualifiée d'inexacte. 
On notera une différence linguistique importante entre les termes précision, imprécision, exactitude et inexactitude. L'imprécision est marquée linguistiquement (par des modifieurs de quantification approximative dans les exemples sous $10 \mathrm{~b}$. et c.), ou est inférée du caractère «rond» de la valeur de référence, auquel cas elle se remarque uniquement (mais jamais univoquement) au niveau du chiffre (a., d., e.) (c'est un cas d'« approximation implicite » chez Adler \& Asnes, 2008 : 1894). Ainsi la précision dans les exemples sous 10 n'est pas marquée explicitement et se laisse seulement lire à déduire du chiffre ${ }^{6}$. L'exactitude et l'inexactitude - et c'est là la grande différence avec les deux autres notions - ne sont pas marquées linguistiquement dans les exemples sous 10, même pas dans le chiffre : elles relèvent de l'évaluation externe de la valeur chiffrée, qui requiert qu'on connaisse la valeur de référence. Tout au plus peut-on dire qu'un chiffre amène avec lui une présomption d'exactitude, tout comme un énoncé asserté amène avec lui une présomption de vérité.

10 Retenons pour le moment qu'une indication chiffrée exacte est, selon ces définitions : $1^{\circ}$ ) un chiffre unique qui correspond à la valeur de référence ou valeur « réelle»; $2^{\circ}$ ) un chiffre qui inclut dans sa zone d'arrondissement la valeur de référence; ou $3^{\circ}$ ) une indication chiffrée qui correspond à une série contigüe ${ }^{7}$ de valeurs qui comprend la valeur de référence. Ce n'est que dans le cas où l'indication chiffrée est exacte que la proposition qui la contient peut être vraie.

Un chiffre précis est un chiffre qui réfère explicitement ou implicitement à une valeur unique et non pas à une fourchette de valeurs.

11 La question qui nous intéresse particulièrement dans le cadre de cette étude est de savoir ce qui se passe si l'on ajoute à une phrase comme 10a. l'adverbe exactement: est-ce que ce dernier marquera alors la précision, l'exactitude ou les deux?

La première chose qu'il faut dire est que la présence de l'adverbe exactement semble exclure la présence d'un modifieur de quantification approximative comme entre ... et .... Cela implique que, des trois cas d'exactitude énumérés dans l'alinéa précédent, seul le premier devra être examiné ici, celui qu'on a dans des exemples comme 11, où nous avons ajouté l'adverbe exactement :

(11) [Le locuteur sait que Marie a 58 ans et dit :]

a. Marie a exactement 58 ans.

b. Marie a exactement 60 ans.

Par la présence de l'adverbe exactement, la lecture d'arrondissement, et donc d'approximation ou imprécision est exclue pour ces exemples. Étant donné que le locuteur de 11 sait l'âge de Marie, il est clair qu'en énonçant b. il ment ou cherche à tromper son interlocuteur. Écartons donc cette phrase, pour nous concentrer sur a, qui est vraie, vu la valeur de référence qu'on connait. Quelle différence y a-t-il alors entre 11a. et 10a.? Pour quelle raison le locuteur peut-il être amené à y ajouter l'adverbe exactement?

11a., à cause d'exactement, a premièrement une lecture d'exactitude. Notre hypothèse est que, par l'ajout de l'adverbe exactement, le locuteur signale qu'il garantit que le chiffre qu'il donne (58) est exact ${ }^{8}$, que celui-ci est conforme, en d'autres termes, à l'âge effectif de Marie. Ce sur quoi le locuteur se fonde pour garantir l'exactitude du chiffre n'est souvent pas spécifié ; le plus souvent il s'agit de l'opération d'obtention de données chiffrées, qui doit être adaptée et fiable (calcul, comptage, inférence...). Une conséquence de ceci est 
que l'adverbe exactement peut être utilisé pour communiquer, indirectement, qu'il y a eu recours à une opération fiable d'obtention de chiffres.

Il est important de souligner que, dans ce type d'exemples, le locuteur est responsable aussi bien de la communication du chiffre que de la communication de la garantie de conformité du chiffre avec la réalité (i.e. avec la valeur de référence) $)^{9}:$ le locuteur est donc à la fois "producteur » et "évaluateur de son propre produit». Ceci est différent par exemple de ce qui se passe avec le marqueur de confirmation effectivement + numéral cardinal, qui signale que le locuteur évalue et confirme un chiffre communiqué par quelqu'un d'autre ${ }^{10}$ :

(12) Marie a effectivement 58 ans.

Exactement, dans des exemples comme 11a., n'est donc pas un marqueur de conformité avec la réalité d'un dire autre (d'un chiffre qui vient d'autrui, Authier-Revuz, 1992, 1993), comme cela est le cas de l'emploi d'exact dans 13, où le producteur du chiffre et l'évaluateur sont des instances différentes:

(13) Ce qu'il a donné comme chiffre est exact.

Ceci n'est pas sans importance, comme nous le verrons $(\$ 4)$ lorsque la phrase contient des marqueurs de source.

Certaines phrases avec exactement ont toutefois aussi une lecture de précision. Prenons, pour le montrer, l'exemple suivant :

(14) [Le locuteur sait que Marie a 60 ans et dit :]

Marie a exactement 60 ans.

17 Dans 14, exactement peut avoir été utilisé par le locuteur pour signaler que le chiffre n'a pas été arrondi, n'est donc pas approximatif, mais précis. Dans cette lecture, l'adverbe équivaut à un "Je vous communique que ce chiffre est précis (i.e. a un degré suffisant de précision) et n'est pas arrondi». Notons qu'une lecture de précision est imaginable également pour la phrase sous 11a. : exactement y signalerait alors par exemple qu'on est le jour de l'anniversaire de Marie et qu'à ses 58 ans d'âge il ne faut ajouter aucun mois, aucun jour, etc. Le taux maximal de précision, souhaité ou suffisant, se détermine par rapport au contexte situationnel, aux intentions communicatives et aux connaissances du monde. Ainsi est-il inhabituel pour un exemple comme 14a., sauf contexte particulier, d'augmenter la précision au-delà des jours, par exemple jusqu'au niveau des heures, des minutes et/ou des secondes. La lecture de précision se rencontre aussi dans les cas où la proposition est en contraste (métalinguistique) avec une autre où l'âge de Marie est décrit de façon approximative :

(15) Marie a non pas « environ » 58 ans mais « exactement » 58 ans.

Dans ce qui précède, nous avons vu qu'une phrase contenant exactement + numéral cardinal (mais sans CE jusqu'ici), à cause de l'interprétation de l'adverbe exactement, peut avoir au moins deux lectures. La question est de savoir si cette double lecture est partout possible ou si une des deux lectures peut être bloquée par le contexte. L'examen d'un grand nombre d'exemples attestés montre d'abord que, dans de nombreux cas, exactement se prête effectivement à deux lectures : 
(16) «Paris. Lundi, 19 février 1951. Il était exactement 22 heures 20. Depuis hier, je n'ai plus vu se soulever ses paupières » (Martin du Gard, 1951, dans Frantext).

(17) « Il tient sa bicyclette de la main droite, il pose sa main gauche sur mon épaule, le visage tourné vers la rue Dupin, il dit : « Regardez. Aujourd'hui il y a exactement quatre semaines jour pour jour que nous nous connaissons » (Duras, 1985, dans Frantext).

(18) « Chaque dessin peut se combiner [...] avec les autres, ce qui fait qu'avec huit dessins on peut en fabriquer quatre... un peu plus de quatre mille, exactement quatre mille quatre-vingt-seize » (Perec, 1979-1981, dans Frantext).

19 Dans 16, une première possibilité de lecture d'exactement est que cet adverbe sert à bloquer la lecture d'arrondissement et donc d'imprécision de 22 heures 20 (il est alors marqueur de précision); une seconde possibilité de lecture est que l'adverbe sert à communiquer que le locuteur garantit que le chiffre correspond réellement à la valeur de référence dans la réalité extralinguistique (il est alors marqueur d'exactitude) ${ }^{11}$.

Dans 17, en lecture de précision, le groupe jour pour jour souligne ce qu'exactement, lui aussi, peut faire dans cette phrase : bloquer la lecture dans laquelle quatre semaines est approximatif au niveau des unités inférieures - les jours; mais exactement peut aussi signaler que le locuteur est en mesure de certifier que l'indication chiffrée de durée est correcte : exactement et jour pour jour jouent alors des partitions séparées.

Dans 18 , c'est la lecture de précision qui s'impose en premier, par l'opposition que crée l'adverbe entre un chiffre imprécis, approximatif, qui précède (un peu plus de quatre mille) et le chiffre précis qui suit ${ }^{12}$; mais une lecture d'exactitude, selon laquelle l'adverbe signale que le locuteur a vérifié et peut garantir la justesse du chiffre donné, ne peut être exclue.

Dans certains exemples, c'est la lecture d'exactitude qui s'impose (plus clairement), rendant la lecture de précision plus difficile ou impossible :

(19) «Un coup d'œil sur ma montre qui marchait comme elle voulait et j'annonçais, péremptoire : "Au quatrième top, il sera exactement sept heures, vingt-sept minutes et quinze secondes" " (Pontalis, 1986, dans Frantext).

(20) "Pour revenir à la position initiale, il faut dix-huit effondrements successifs, dix-huit périodes de 24.000 ans... Cela fait exactement 432.000 ans, le chiffre que la grande tradition hindoue a toujours donné pour la durée du kaliyuga » (Abellio, 1946, dans Frantext).

(21) « Roanne rejette par jour en moyenne 30773 kilos d'ordures ménagères. J'en conclus que cette ville doit avoir exactement 38467 habitants » (Tournier, 1975, dans Frantext).

(22) «En analysis sitûs les expériences approchées peuvent suffire pour donner un théorème rigoureux et, par exemple, si l'on voit que l'espace ne peut avoir ni deux ou moins de deux dimensions, ni quatre ou plus de quatre, ou [sic] est certain qu'il en a exactement 3 , car il ne saurait en avoir 2 et demi ou 3 et demi » (Poincaré, 1905, dans Frantext).

(23) « Je pense aux saints Jean et Paul, dont c'est le jour, en attendant l'heure de la messe. [...] J'aime ces martyrs étrangement privilégiés, en si petit nombre, qui sont nommés tous les matins au sacrifice dans le monde entier. Ils sont exactement trente-neuf » (Bloy, 1900, dans Frantext).

Dans 19, la précision/imprécision n'est pas vraiment en jeu - on sait que l'horloge parlante ne va pas aller au-delà des secondes ${ }^{13}$, pas besoin donc de signaler que l'on a omis les fractions de seconde ; c'est donc l'exactitude de l'indication horaire qui est importante et qui est garantie par le locuteur au moyen de l'adverbe exactement. 
Dans 20, le chiffre a été obtenu par une simple multiplication. Puisque la seule mention du chiffre, sans l'adverbe, aurait très bien pu suffire, la question se pose de savoir quelle est la raison d'être de l'adverbe. Peu probable qu'il serve à signaler ici le nonarrondissement: les chiffres sur lesquels est fondée la multiplication sont eux-mêmes ronds (ou arrondis), ce qui fait que la multiplication ne peut que donner un chiffre rond. Il nous semble donc qu'exactement y sert à souligner que le chiffre est exact, correct ( «je peux garantir la justesse de ce chiffre sur la base du calcul que j'ai fait »).

Une analyse analogue en lecture d'exactitude vaut pour 21 , où la lecture de précision rencontre la difficulté supplémentaire du chiffre non rond.

Dans 22, il est clair que le locuteur peut sans aucun problème garantir l'exactitude du chiffre, par inférence, après avoir exclu toutes les autres possibilités. Il n'est pas question de précision ici. Dans 23, le locuteur peut garantir l'exactitude du chiffre sur la base d'un comptage fait à partir de l'énumération des saints lors du service religieux.

Lorsqu'exactement a la lecture d'exactitude, il peut être paraphrasé, on l'a vu, par « pas plus et pas moins ». Dans certains cas toutefois - et cela tient aux caractéristiques des numéraux et à l'effet du contexte -, l'exactitude sera paraphrasée uniquement par « et pas moins » :

(24) Windows met exactement 15 minutes à démarrer. Je vois l'icône réseau avec le "rond" d'initialisation du réseau qui tourne pendant exactement 15 minutes ${ }^{14}$.

Il y a un effet pragmatique qui accompagne une telle interprétation asymétrique, à savoir que cela suggère une évaluation quantitative en termes de « peu » ou de « beaucoup ».

\section{La valeur du conditionnel épistémique}

Le conditionnel épistémique appartient à l'un des trois grands groupes d'emplois du conditionnel ${ }^{15}$. Il sert à régler, le plus souvent, ce que H.Kronning appelle la "responsabilité énonciative» du locuteur en qualifiant tel ou tel aspect du savoir communiqué : son degré de certitude, sa provenance ou sa (non-)prise en charge par le locuteur au moment de l'énonciation.

Les observations faites au début de cette étude sur la fréquence d'apparition de la combinaison conditionnel + exactement + numéral cardinal ne valent d'ailleurs pas pour les deux autres groupes de conditionnels (le conditionnel temporel - cf. 25 - et le conditionnel d'éventualité - $c f .26$ ), qui se combinent, sans difficulté et fréquemment, avec exactement + numéral cardinal :

(25) «Il rappelait récemment à l'un de ses proches qu'il aurait exactement quatre vingt dix ans en mars 2004, date prévue pour la tenue du VII ${ }^{\text {ème }}$ congrès. Il n'avait plus tellement envie de devoir s'y expliquer devant ses camarades ${ }^{16}$.

(26) «Si seul, le hasard intervenait, étant donné que le dé comporte six faces, il y aurait exactement une chance sur six pour qu'une face quelconque tombe tous les six lancers $»^{17}$.

Le CE a fait l'objet de plusieurs études de détail en linguistique. Selon P. Dendale (1993), trois traits sémantiques sont régulièrement associés par les linguistes et les grammairiens à cet emploi du conditionnel : un trait évidentiel (l'emprunt d'un contenu propositionnel à 
autrui), un trait modal (l'incertitude) et un trait aléthique (la non-prise en charge de l'information).

Selon quelques analyses plus récentes de H. Kronning (2002, 2005, 2012), le conditionnel serait un marqueur grammatical dit " mixte », qui signale toujours deux choses par luimême : l'emprunt d'un contenu propositionnel à autrui et le refus de prise en charge de ce contenu. Cela veut dire que, pour H. Kronning, dans un énoncé comme 27,

(27) «Le rédacteur en chef du Mirror, Piers Morgan, se déclare "absolument persuadé" de l'authenticité des photos que le journal aurait payées 5000 livres (7500 euros) à chacun des deux soldats qui les lui ont fournies " (Le Monde).

\section{Conditionnel épistémique + exactement + numéral cardinal}

Examinons maintenant la valeur de l'adverbe exactement combiné au CE dans une même phrase. Nous le ferons à partir de quelques-uns des rares exemples attestés que nous en avons trouvés pour le moment.

\subsection{Première série : exemples avec un second marquage de la source}

Commençons par dire que l'adverbe exactement, placé dans un contexte d'emprunt, peut se prêter, théoriquement, à deux séries de lectures : l'une où c'est le locuteur d'origine 
qui avait utilisé l'adverbe dans l'énoncé source et l'autre où c'est le locuteur final qui a rajouté l'adverbe à l'énoncé emprunté. Examinons d'abord le premier cas :

(30) « 14 juil. 2006 - Selon les responsables d"“Otpor" de Danilovgrad, le mouvement compterait "exactement 777 membres", mais un policier de la ville affirme qu'en fait, "ils tiendraient tous dans une voiture" $»^{19}$.

Dans cet exemple, exactement fait partie de ce que J. Authier-Revuz $(1992,1993)$ appelle un «ilot textuel», un petit bout de citation du discours d'origine. Théoriquement, exactement, placé dans le discours direct et dans la modalisation autonymique, peut avoir les deux fonctions décrites au $\$ 2$ : signaler la précision et signaler l'exactitude. Dans l'exemple 30, la lecture de précision est toutefois exclue parce que le chiffre lui-même est précis et il n'est pas non plus en opposition cotextuelle avec un chiffre imprécis, approximatif qui le précéderait. En revanche, l'adverbe exactement peut signaler ici que le locuteur source garantit que le chiffre est conforme à la réalité (lecture d'exactitude). Ce qui est important à souligner ici, c'est que c'est alors le locuteur source et non le locuteur final qui garantit l'exactitude du chiffre.

Le CE dans cet exemple a les valeurs décrites sous $\$ 3$ : emprunt (doublement marqué dans cette phrase) et refus de prise en charge par le locuteur final de la proposition empruntée. Dans cet exemple, le locuteur final refuse de prendre position par rapport à la vérité de la proposition et à l'exactitude du chiffre. Dans l'exemple suivant, le locuteur final précise pourquoi il ne peut pas prendre en charge l'information chiffrée et pourquoi il la transmet telle quelle (On les a pas comptées) :

(31) « Puisque dans ce pays il faut obtenir un permis (et il y a des quotas) pour bivouaker en montagne, nous avons du prendre le plus raide de ces chemins (les autres étant complets bien sur...). Parait qu'il y aurait exactement 101 épingles dans le chemin... On les a pas comptées... » ${ }^{20}$.

Ce même exemple illustre une deuxième situation d'emploi d'exactement dans des contextes d'emprunt: lorsque l'adverbe ne figurait pas dans le texte source - celui-ci contenait alors seulement le chiffre - et que c'est le locuteur final qui l'a ajouté. C'est, nous semble-t-il, le cas dans des exemples comme 32 et 33, où le locuteur final a emprunté la donnée chiffrée à des statistiques, simples listes de chiffres contenant rarement des adverbes comme exactement :

(32) «Selon les derniers chiffres du premier trimestre 2005, la France compterait exactement 1.884.479 lignes en dégroupage partiel, et 152.189 en dégroupage total. Et l'ARCEP estime à 500000 le nombre de lignes totalement dégroupées à la fin de l'année $»^{21}$.

(33) « 1 mai 2006 - Selon l'article, il y aurait en Éthiopie exactement 86762 fermiers cultivant le café Shirkhina pour Starbuck $»^{22}$.

Dans ces exemples, il n'est pas possible, vu la valeur du CE (marquer le refus de prise en charge par le locuteur final), de voir dans exactement un élément qui signalerait que ce même locuteur final garantirait l'exactitude du chiffre et donc la vérité de la proposition. Cela serait contradictoire. Mais comme ces phrases sont attestées et paraissent en plus tout à fait normales, il faut que l'adverbe y ait une autre valeur. Laquelle? Nous voyons plusieurs possibilités.

Une première possibilité est que l'adverbe, ajouté par le locuteur final, serve à signaler premièrement que le chiffre se donnait (explicitement) pour exact dans l'énoncé source, ou 
qu'il y était investi d'une " présomption d'exactitude », ou deuxièmement qu'il avait été obtenu par le locuteur source à la suite d'une opération fiable d'obtention de données chiffrées, opération mentionnée ou suggérée dans l'énoncé source. Le locuteur final " paraphrase » alors l'exactitude du chiffre provenant de l'énoncé source, sans la garantir lui-même: il est responsable du choix d'utiliser le mot exactement, pas responsable de l'exactitude du chiffre.

Une deuxième possibilité, moins évidente et moins fréquente, est que l'adverbe, ajouté par le locuteur final, serve à attirer l'attention sur la précision (ou sur l'étrangeté de la précision) des chiffres, par exemple en contraste avec celui qui croirait que seuls des chiffres approximatifs pourraient être donnés.

Accessoirement, l'adverbe peut aussi marquer l'étonnement du locuteur final par rapport à la possibilité d'obtenir, en la matière, des chiffres exacts et/ou précis.

Le CE, dans les deux cas, joue son rôle de marqueur de refus de prise en charge, dans un sens faible ou dans un sens fort du terme. Le sens faible signifie que le locuteur final a présenté les chiffres "tels quels", sans nullement laisser transparaitre sa position personnelle sur leur exactitude - si tant est qu'il en a une - tout en signalant (par paraphrase) que pour le locuteur source ils sont bel et bien exacts/précis. Le sens fort de refus de prise en charge signifie que le $\mathrm{CE}$ contribue à renforcer l'attitude d'étonnement du locuteur final - suggérée pragmatiquement par l'adverbe exactement ajouté par ce dernier - quant à l'exactitude du chiffre ou à la possibilité d'obtenir des chiffres exacts ou précis. Ce sens fort peut même aller jusqu'à la mise en doute de l'attitude du locuteur final. C'est ce que nous montrons dans la section suivante.

\subsection{Deuxième série : exemples avec un marquage supplémentaire de prise de distance}

Prenons l'exemple suivant :

(34) «Selon le CESNUR, il y aurait exactement aujourd'hui, de par le monde, quatre sociologues qui admettraient l'existence d'un lavage de cerveau dans les sectes, tous les autres la niant. C'est ignorer le nombre de psychologues et psychiatres, au moins aussi titrés dans leur spécialité que les experts du CESNUR - sociologues et avocats - qui trouvent, après étude de cas concrets, que le modèle "lavage de cerveau" (ou " réforme de la pensée") rend bien compte, avec les nuance nécessaires, de ce qui s'opère chez les nouvelles recrues, comme de leurs difficultés de se libérer $»^{23}$.

La fin de cet exemple (C'est ignorer...) explicite les doutes sérieux du locuteur final quant à l'exactitude du chiffre avancé par le locuteur source. L'adverbe exactement, ajouté ou non par le locuteur final, souligne que pour le locuteur source (le CESNUR) - et lui seul -, il est exact de dire qu'il y a quatre sociologues (« et pas plus ») ${ }^{24}$ qui admettent l'existence d'un lavage de cerveau dans les sectes.

Un autre exemple attesté, fort intéressant est le suivant :

(35) « Surprenant chiffre : 367 . Il y aurait exactement, à l'unité près 367 porteuses de voile intégral en France. On est d'abord surpris d'une telle précision : toutes les femmes portant le niqab ou la burqa seraient-elles suivies et fichées par la police? En ce cas c'est très inquiétant. Autre interrogation, c'est la modestie du chiffre donné par une soi-disant fuite des Rgs. Si le voile intégral, qui n'est aucunement prescrit en Islam, est, on le sait, un phénoméne 
fortement minoritaire, le nombre est si dérisoire qu'il me parait fabriqué de toutes piéces pour enterrer le débat. Comme le dit le député vert François de Rugy: "ces chiffres sont assez ridicules. J'ai le plus grand respect pour le travail des policiers, mais là je crois que les renseignements généraux nous font un peu croire n'importe quoi. N'importe quel Français se promenant dans la rue peut voir que le phénomène de la burqa ou du niqab est beaucoup plus important que quelques centaines de cas à l'échelle de la France. Ça ne sert à rien de vouloir nier le problème.". [...] Mais quoi qu'il en soit une question me reste en tête: comment les Rgs sont-ils arrivés à ce chiffre de $367 ? »^{25}$. été rajouté par le locuteur final. Nous partirons du second cas, qui nous semble le plus probable, vu les autres éléments de la phrase. Exactement peut avoir été rajouté pour attirer l'attention sur l'exactitude du chiffre 367. L'adverbe est suivi du groupe à l'unité près, précision à première vue redondante et inutile, mais qui contribue à donner à l'adverbe une lecture ironique de rejet de l'exactitude, de la conformité à la réalité. Cette lecture « ironique» est confirmée par les nombreuses indications dans la suite du texte, qui mettent en cause la possibilité de précision (surpris d'une telle précision), la possibilité d'exactitude (comment les Rgs sont-ils arrivés à ce chiffre ?) ou, pour finir, l'exactitude même (la modestie du chiffre; ces chiffres sont assez ridicules; font un peu croire n'importe quoi; le phénomène est beaucoup plus important que quelques centaines de cas...).

Le CE se sent très à l'aise dans ce type de contexte. Il a vraisemblablement été rajouté par le locuteur final, car il peut difficilement avoir figuré dans l'énoncé source. Il marque le refus du locuteur de prendre en charge l'information (et donc le chiffre) - refus de prise en charge au sens fort (cf. supra (§ 4.1.) - et est parfaitement en phase avec tous les éléments explicites dans cet extrait et la lecture ironique d'exactement.

Une autre version du même sujet sur le voile intégral, trouvée également sur Internet, explicite, différemment, les raisons de la difficulté d'obtenir un chiffre exact en la matière. Le conditionnel y remplit pleinement ses fonctions d'emprunt d'information à autrui et de refus de prise en charge de l'information (au sens fort) :

(36) «Il y aurait exactement 367 femmes en France qui porteraient le voile intégral, soit le niqab ou la burqa. C'est proprement phénoménal. 367 ! Pas une de plus, pas une de moins. Ce sont les services du ministère de l'Intérieur qui ont compté ces dames. Ils sont tellement doués qu'ils ont su discerner dans le lot pour les décompter celles et ceux qui s'étaient déguisés le jour du carnaval, et ils ont rajouté au cheptel celles qui ne sortent de chez elles qu'à la nuit tombée $»^{26}$.

Un autre exemple de ce type dans notre corpus est 37 :

(37) "Selon "Migrations and remittances factbook 2008", une étude que vient de publier la Banque Mondiale, il y aurait exactement (!) 1.213 .042 émigrés maliens à travers le monde, soit $9 \%$ de la population totale malienne (14 millions) $»^{27}$.

41 L'adverbe exactement y est suivi d'un point d'exclamation, qui traduit l'étonnement du locuteur final quant au chiffre, plus précisément quant à sa possibilité de précision et (donc) d'exactitude - à cause du comptage extrêmement difficile dû au grand nombre d'individus à compter et au fait que la situation démographique, à cette échelle, évolue sans doute plus vite que le temps requis pour le recensement. La valeur d'exactement et du CE sont comparables à celles qu'ils ont dans les trois exemples précédents.

Pratiques, $167-168 \mid 0000$ 


\subsection{Troisième série : exemples sans marquage supplémentaire}

45 été rajouté par le locuteur final. S'il était présent dans l'énoncé source, il y permettait au locuteur source d'attirer l'attention sur l'exactitude du chiffre, soit pour souligner que l'information provenait d'une opération fiable d'obtention du chiffre, soit pour laisser entendre une évaluation quantitative du chiffre en termes de «c'est beaucoup » ou " c'est peu » (cette dernière possibilité est exclue ici, car contredite par le cotexte droit). S'il a été ajouté par le locuteur final, il sert à expliciter la présomption d'exactitude qu'emporte avec lui le chiffre dans l'énoncé source, pour souligner donc que le chiffre avait été donné pour exact-pour-le-locuteur-source, ou pour indiquer que dans l'énoncé source le chiffre était fondé sur une opération fiable d'obtention de chiffres, ou encore pour laisser entendre une évaluation en termes de " c'est beaucoup » de la part du locuteur final.

Dans cet exemple, le CE a sa valeur normale de marqueur d'emprunt, signalant que l'information chiffrée à été empruntée à un locuteur source et empêchant ainsi qu'on l'interprète comme provenant d'une opération de comptage effectuée par le locuteur final. $\mathrm{Au}$ niveau de la valeur de refus de prise en charge du $\mathrm{CE}$, cet exemple pose problème quand on suit l'analyse de H. Kronning. Ou bien on dit que le CE exprime un refus de prise en charge au sens faible, tel que décrit plus haut ( Je vous présente l'information "telle quelle" »), mais alors on comprend mal comment le locuteur peut y enchainer le contenu de la relative en ce qui à l'indicatif présent (et pas au conditionnel); ou bien on considère que la proposition au CE se base sur une attitude de certitude du locuteur final - auquel cas d'ailleurs exactement pourrait signifier la garantie par le locuteur final - et dans ce cas, 
le CE, tout en gardant sa valeur de non-prise en charge (mais dans quelle interprétation?) aurait, selon l'analyse de H. Kronning (2012: 87), une fonction d'organisation textuelle, qui consiste à « évoquer un point de vue d'autrui, sans l'endosser ${ }^{29}$, afin de pouvoir, dans la suite de son discours, l'infirmer [...] ou le confirmer ». Et là, le problème est, premièrement, que la suite (ce qui...) n'est ni une confirmation, ni une infirmation de ce qui précède, mais une conclusion qui, pour être valable et avoir du sens, doit attribuer aux arguments le statut de vérité ; et, deuxièmement, la question se pose alors de savoir à quel type de refus de prise en charge on peut bien avoir à faire: forte («doute») ? certainement pas ; faible (« indécision, ignorance »)? difficile à rimer aussi avec la suite de l'extrait. En résumé, le problème que met au jour cet exemple est un problème pour l'analyse de H. Kronning du CE.

\section{Conclusion}

La question qui sous-tendait cette étude était de savoir pourquoi il est si difficile de trouver des exemples attestés de l'adverbe exactement suivi d'un numéral cardinal en combinaison avec le conditionnel épistémique de reprise (CE), alors que ce dernier se combine sans aucun problème avec des modifieurs de quantification approximative.

Pour essayer de trouver une réponse à cette question, nous avons décrit la valeur sémantique et les fonctions d'exactement suivi d'un numéral cardinal - ce qui n'avait pas encore été fait jusqu'ici, que nous sachions. Cette description a montré que l'adverbe exactement est utilisé le plus souvent pour communiquer que le locuteur garantit que le chiffre est exact, ou sinon pour signaler qu'un chiffre rond n'est pas arrondi et est donc précis.

Placé dans un contexte où apparait un $\mathrm{CE}$, qui signale l'emprunt et le refus de prise en charge par le locuteur, certaines fonctions d'exactement - parmi les plus importantes sont bloquées. Ainsi ne peut-il plus signaler que le locuteur final garantit l'exactitude de l'information, car cela serait contradictoire avec la valeur de non-prise en charge du CE. Les cas où l'adverbe n'entre pas en contradiction avec la valeur du CE sont ceux où le locuteur final cite le locuteur source (c'est rare), où il traduit la présomption d'exactitude du chiffre dans l'énoncé source (les cas où il a besoin de faire cela ne sont pas fréquents non plus) ou bien où il indique le caractère précis du chiffre provenant de l'énoncé source (et cela est limité aux seuls chiffres ronds). Restent les cas où le locuteur final prend clairement ses distances par rapport aux prétentions d'exactitude du chiffre dans le message d'origine.

\section{BIBLIOGRAPHIE}

Au final, la combinaison n'est pas impossible, mais les situations où elle peut se rencontrer sont plutôt exceptionnelles, ce qui explique qu'on en ait trouvé relativement peu d'exemples attestés. Ce n'est donc certainement pas une exclue de la langue, mais une rareté, pour les raisons indiquées. 
ABOUDA, L. (1997). Recherches sur la syntaxe et la sémantique du conditionnel en français. Thèse de doctorat, Université Paris 7.

ADLER, S. \& ASNES, M. (2008). « Approximation par arrondissement : le cas de quelques quantifieurs prépositionnels ». In: Durand, J., Habert, B. \& Laks, B. (éds), Congrès mondial de linguistique française - CMLF 08. Paris : Institut de Linguistique Française, p. 1891-1903.

- (2014). «Quantification approximative et quantification floue: essai de précision ». In : BatZeev Shyldkrot H., Adler, S. \& Asnes, M. (éds), Précis et imprécis: études sur l'approximation et la précision. Paris : Champion, p. 25-42.

AUTHIER-REVUZ, J. (1992). « Repères dans le champ du discours rapporté (I) ». L'Information grammaticale 55, p. 38-42.

- (1993). « Repères dans le champ du discours rapporté (II) ». L'Information grammaticale 56, p. $10-15$.

DENDALE, P. (1993). « Le conditionnel de l'information incertaine : marqueur modal ou marqueur évidentiel ? ». In: Hilty, G. (éd.), Actes du XX Congrès international de linguistique et philologie romanes, université de Zurich (6-11 avril 1992). Tübingen : Francke, t. 1, p. 165-176.

- (2012). «Le conditionnel "épistémique”, marqueur de modalisation en discours second: éléments d'archéologie grammaticale ». In: Branca-Rosoff, S. et al. (éds), L'Hétérogène à l'œuvre dans la langue et les discours. Hommage à Jacqueline Authier-Revuz. Paris : Lambert-Lucas, p. 229-248.

KRONNING, H. (2002). « Le conditionnel “épistémique” : médiation et modalisation épistémiques ». Romansk Forum 16, 2, p. 561-575.

- (2005). «Polyphonie, médiation et modalisation : le cas du conditionnel épistémique ». In: Bres, J. et al. (éds), Dialogisme, polyphonie : approches linguistiques. Bruxelles : De Boeck/Duculot, p. 297-312.

- (2012). «Le CE : propriétés et fonctions discursives ». Langue française 173, p. 83-97.

SCHAPIRA, C. (2010). « Précision ou, plus exactement, focalisation discursive ». L'Information grammaticale 125, p. 17-21.

WILMET, M. (2010). Grammaire critique du français. Paris : Duculot.

\section{NOTES}

1. En ligne: http://www.legrandsoir.info/les-etudiants-du-chili-sont-dans-la-rue-desaffrontements-et-des-centaines-d-arrestations.html. Consulté le 16/02/16.

2. En ligne : http://www.babla.fr/francais-allemand/compterait. Consulté le 16/02/16.

3. Elles se fondent sur leur emploi en sciences exactes.

4. Il y a un facteur dont les auteurs font abstraction, c'est que l'indication 58 ans est nécessairement imprécise quant au nombre de mois, jours, heures, etc. qui s'ajoutent aux années. Ce n'est que quand on fait abstraction de cela qu'on peut effectivement qualifier a. de précis.

5. La seconde lecture dans les deux derniers exemples est celle d'un arrondissement, qui correspond aux cas illustrés respectivement par b. et c.

6. À condition bien sûr de faire abstraction des unités de niveau inférieur (mois, jours, décimales) d'un chiffre.

7. Dans les exemples contenant un modifieur de quantification approximative où une pluralité de valeurs possibles est donnée pour l'âge de Marie, les valeurs sont toujours contigües: elles constituent des séries continues (et ce contrairement à la métaphore de la cible, que les auteurs 
donnent pour expliquer les termes, et dans laquelle les valeurs peuvent se trouver dispersées dans toutes les dimensions autour du centre de la cible).

8. Par cette garantie, le marqueur exactement pourrait être considéré comme un marqueur explicite de prise en charge par le locuteur de la vérité de la proposition.

9. Ceci est également le cas dans la lecture «exactitude » d'exemples comme: Peux-tu me dire 1 'heure exacte? Ce qu'on demande à l'interlocuteur est de constater l'heure et de s'assurer que l'heure communiquée est bien l'heure réelle.

10. Ce « quelqu'un d'autre " pouvant être le locuteur même, mais à une autre époque.

11. Ce que 16 a de subtil est que le temps passé d'était crée une distance entre le moment où le locuteur a vécu les évènements et le moment où il les raconte. La garantie de l'exactitude de l'heure peut donc dater aussi bien de l'époque (je l'avais vérifié à l'époque) que du moment d'énonciation (je viens de le vérifier et le certifie en ce moment même).

12. La meilleure glose d'exactement y est plus précisément, dont C. Schapira (2010:18) a montré qu'il a une lecture de restriction.

13. Ne fût-ce parce que le troisième top prend largement plus de temps qu'une unité inférieure à la seconde.

14. En ligne: http://forum.hardware.fr/hfr/WindowsSoftware/Windows-7-seven/exactementminutes-demarrer-sujet_328958_1.htm. Consulté le 15/02/16.

15. Signalons au passage que les deux autres grands groupes d'emplois du conditionnel sont le conditionnel temporel - illustré par 25 et 26 - et le conditionnel d'éventualité - illustré par 27 et 28. Nous laisserons de côté ces deux groupes d'emplois :

(i) Les Argentins pensaient que les Anglais se contenteraient de faire un peu de bruit pour la forme (Nouvel Observateur).

(ii) Bonaparte désarma celui qui deviendrait bientôt son rival et son adversaire (Bainville, cité par Damourette \& Pichon V:§1842).

(iii) Si cette guerre n'était pas si tragique, je serais tenté de l'appeler la guerre des «parrains »( Nouvel Observateur).

(iv) Une telle réaction serait désastreuse.

16. En ligne : http://www.maghress.com/fr/marochebdo/116345. Consulté le 27/02/16.

17. En ligne : http://www.pnl-nlp.org/courses/ebooks/page.php?bid=287\&pageid=4431. Consulté le 09/10/15.

18. En ligne: https://www.facebook.com/voixdujura/posts/10150138793960859. Consulté le 27/02/16.

19. En ligne : http://www.perso.ch/troubles/albanie.htm. Consulté le 27/02/16.

20. En ligne: http://pauloetpaulette.hautetfort.com/archive/2010/10/18/rencontre-avec-unours.html. Consulté le 27/02/16. L'orthographe du site a été conservée.

21. En ligne: http://misstics.canalblog.com/archives/2005/07/19/663284.html. Consulté le 27/02/16.

22. En ligne : http://monastique.blogspot.be/2006/05/le-cafe-equitable-comme-temoin-des.html . Consulté le $27 / 02 / 16$.

23. En ligne : http://www.prevensectes.com/lobbies1.htm. Consulté le 27 /02/16.

24. Il nous semble que, dans ce cas, on a affaire aussi à une asymétrie dans ce que suggère évaluativement exactement.

25. En ligne: http://www.romainblachier.fr/2009/07. Consulté le 27 /02/16. L'orthographe du site a été conservée.

26. En ligne : http://amertume.over-blog.com/article-34370799.html. Consulté le 27 /02/16.

27. En ligne: http://www.mybloglog.com/buzz/community/geographientic/ consulté 18/1/2010, page plus accessible. Consulté le 15/05/14.

28. En ligne: http://www.pokemontrash.com/1318-Rumeurs-Pokemon-Black-_-White.html. Consulté le 27 /02/16. 
29. La formulation ne suggère nullement que « refus de prise en charge » est à prendre au sens fort; le terme "refus " nous semble même fort pour désigner quelque chose qui n'est autre qu'une non-prise de position.

\section{RÉSUMÉS}

Cet article porte sur une combinaison d'éléments, dont on pourrait supposer qu'elle serait exclue du point de vue théorique : le conditionnel épistémique + exactement + un numéral cardinal. En effet, on pourrait s'attendre à un conflit entre, d'une part, le sémantisme du marqueur exactement , dont la principale valeur est de signaler que le locuteur garantit que le chiffre donné est exact et/ou précis, et, d'autre part, le conditionnel épistémique (CE), qui signale le refus de prise en charge de ce même chiffre par le locuteur final. Or, la combinaison est bel et bien attestée dans certains corpus (autres que Frantext), toute rare qu'elle demeure. Ces exemples attestés mettent en doute le conflit théorique et invitent à s'interroger sur les conditions qui rendent la combinaison possible.

Pour trouver une explication à ce phénomène, nous décrivons d'abord le sémantisme de l'adverbe (marqueur d'exactitude et de précision, selon les cas) et examinons l'effet, sur l'interprétation de l'adverbe et sur ses possibilités d'emploi, de la présence dans la phrase d'un conditionnel épistémique, marqueur de refus de prise en charge et de reprise d'information à autrui.

La conclusion de l'étude est que l'adverbe exactement n'entre pas en conflit avec la valeur du CE lorsque : le locuteur « final » cite le locuteur source, lorsqu'il traduit la présomption d'exactitude du chiffre de l'énoncé source, ou lorsqu'il indique le caractère précis du chiffre provenant de l'énoncé source. La combinaison n'est donc pas une exclue de la langue, mais les situations où elle peut se rencontrer sont très contraintes, ce qui explique qu'on en trouve relativement peu d'exemples authentiques.

This article focuses on a combination of language units in French that one could expect, from a theoretical point of view, to be excluded: the epistemic conditional + exactement + cardinal numeral. One could indeed think that there would be a conflict between, on the one hand, the semantics of the marker exactement, whose main value is to report that the final speaker guaranties that the given number is accurate and/or precise, and on the other hand, the epistemic conditional (CE), which signals that the information was taken from someone else and that the speaker refuses to commitment himself to the correctness of that number. The combination however is attested in some corpora (other than Frantext), be it scarcely. The attested examples cast doubt about the theoretical conflict and invite to examine the conditions that make the combination possible.

In order to find an explanation for this phenomenon we describe the semantics of the word (marker of accuracy or precision, depending on the contexts) and examine the effect, on the interpretation of the adverb and on its possibilities of use, of the presence of an epistemic conditional in the sentence, marker of commitment refusal.

The conclusion of the study is that the adverb does not conflict with the value of the CE: when the "final" speaker quotes the "source speaker", when he translates the presumption of correctness of the number in the source utterance, or when he wants to indicate that the number in the source utterance is precise. The combination is thus not excluded, but the situations where 
it can be found are very constrained, which explains why one indeed finds so few authentic examples of it.

INDEX

Mots-clés : conditionnel épistémique de reprise, marqueurs d'approximation, expression de l'exactitude, expression de la précision, exactement, numéral cardinal

Keywords : epistemic (reportative) conditional, markers of approximation, expressions of exactitude, expressions of precision, cardinal numeral

\section{AUTEURS}

\section{PATRICK DENDALE}

Université d'Anvers

DIANA ANDREI

EHESS (Paris) 